\title{
Discrete wavelet and modified PCA decompositions for postural stability analysis in biometric applications
}

\author{
Dhouha Maatar ${ }^{1,2}$, Regis Fournier ${ }^{1}$, Zied Lachiri $^{2}$, Amine Nait-Ali ${ }^{1}$ \\ ${ }^{1}$ Université Paris-Est Créteil, Laboratoire Images, Signaux et Systèmes Intelligents, Créteil, France; \\ ${ }^{2}$ ENIT, Laboratoire Traitement du Signal de l'Image et Reconnaissance de Formes, Tunis, Tunisie. \\ Email: doha.maatar@gmail.com; rfournier@u-pec.fr; zied.lachiri@enit.rnu.tn; naitali@u-pec.fr
}

Received 3 January 2011; revised 15 March 2011; accepted 29 April 2011.

\section{ABSTRACT}

The aim of this study is to compare the Discrete wavelet decomposition and the modified Principal Analysis Component (PCA) decomposition to analyze the stabilogram for the purpose to provide a new insight about human postural stability. Discrete wavelet analysis is used to decompose the stabilogram into several timescale components (i.e. detail wavelet coefficients and approximation wavelet coefficients). Whereas, the modified PCA decomposition is applied to decompose the stabilogram into three components, namely: trend, rambling and trembling. Based on the modified PCA analysis, the trace of analytic trembling and rambling in the complex plan highlights a unique rotation center. The same property is found when considering the detail wavelet coefficients. Based on this property, the area of the circle in which 95\% of the trace's data points are located, is extracted to provide important information about the postural equilibrium status of healthy subjects (average age $31 \pm 11$ years). Based on experimental results, this parameter seems to be a valuable parameter in order to highlight the effect of visual entries, stabilogram direction, gender and age on the postural stability. Obtained results show also that wavelets and the modified PCA decomposition can discriminate the subjects by gender which is particularly interesting in biometric applications and human stability simulation. Moreover, both techniques highlight the fact that male are less stable than female and the fact that there is no correlation between human stability and his age (under 60).

Keywords: Approximation Wavelet Coefficients; Detail Wavelet Coefficients; Discrete Wavelet Analysis; PCA Decomposition; Phase; Rambling, Stabilogram, Trembling; Trend, Biometrics

\section{INTRODUCTION}

Analysis of postural sway is of great interest because it can be used to identify changes in balance control mechanisms. Basically, these changes depend on the age of the person and they occur due to some diseases.

Except the aging, balance control can also be affected by gender and different sensory systems, including vestibular, visual, and proprioception systems [1-3].

The postural sway is generally quantified by displacement of the Center of Pressure (CoP) over the time. This displacement is performed by standing in static position on a platform based on magnetic field $[4,5]$.

The study of postural control sway is performed by analyzing the stabilogram which is the representation of COP's displacement in anteroposterior (AP) and mediolateral (ML) direction. In this paper, we consider the Gravity Center (GC), instead of COP as described in [4-7].

Several studies showed that the stabilogram is considered as non-stationary signal, produced by a non-linear system [8]. To analyze such a signal, numerous techniques have been proposed. For instance, the Empirical Mode Decomposition (EMD) has been proposed. The EMD allows an efficient extraction of intrinsic mode functions, called (IMF) [6,9-11]. Standard Fourier transform has also been used to analyze human posture stability. In particular, in has been used to highlight the correlation between the fear of falling and strategies produced by human postural control [12].

On the other hand, wavelet analysis [13] has been employed in numerous studies for the purpose to determine both short-term and long-term diffusion coefficients from the stabilogram diffusion control [14-17]. It has been used also to discriminate chronic ankle instability [18].

In this work, the stabilogram is analyzed using both wavelet decomposition and the mPCA decomposition (mPCA) $[4,6]$. The main goal of this study is to analyze, 
using these tools, the effect of: 1) vision on the human postural control; 2) stabilogram direction; 3) age; 4) gender. For this purpose we have constructed a database of stabilograms, recorded from healthy voluntary subjects.

This paper is organized as follows: in Section 2, we describe the experimental protocol. Afterwards, the mPCA and wavelet decomposition methods are presented in Section 3. Finally, our results and discussions are provided in Section 4.

\section{MATERIALS AND MEASURES}

Experimental measures are recorded while an individual stand upright on a electromagnetic platform [4]. After performing a calibration and correction phases on these measures, we obtained the CG displacement in the horizontal plane.

The representation of this CG displacement in medio- lateral (ML) or anterioposterior (AP) directions is called the stabilogram [6] as shown in Figure 1.

After the calibration phase, the measures are achieved with subjects standing in an orthostatic position during 30 seconds. Each recorded signal is sampled at $60 \mathrm{~Hz}$. The measures are then evaluated for twenty five healthy subjects, including 8 females and 17 males aged between 19 years and 42 years.

- The first measure is achieved by keeping foot outspread and opened eyes fixing a point placed on the wall in front of the subject (PE_YO),

- The second measure is preformed in the situation of tighten foot and opened eyes (PS_YO),

- $\quad$ The third measure is achieved by considering outspread foot and closed eyes (PE YF),

- The last measure considers tightened foot and closed eyes (PS_YF).

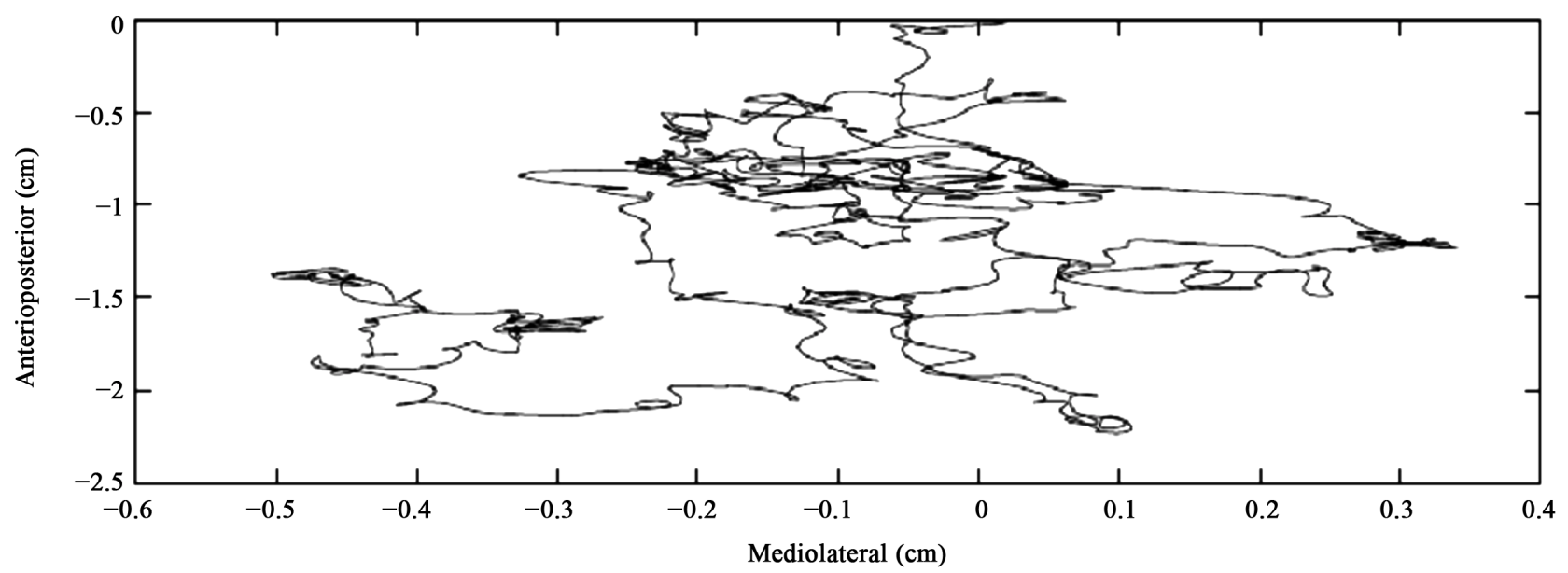

(a)

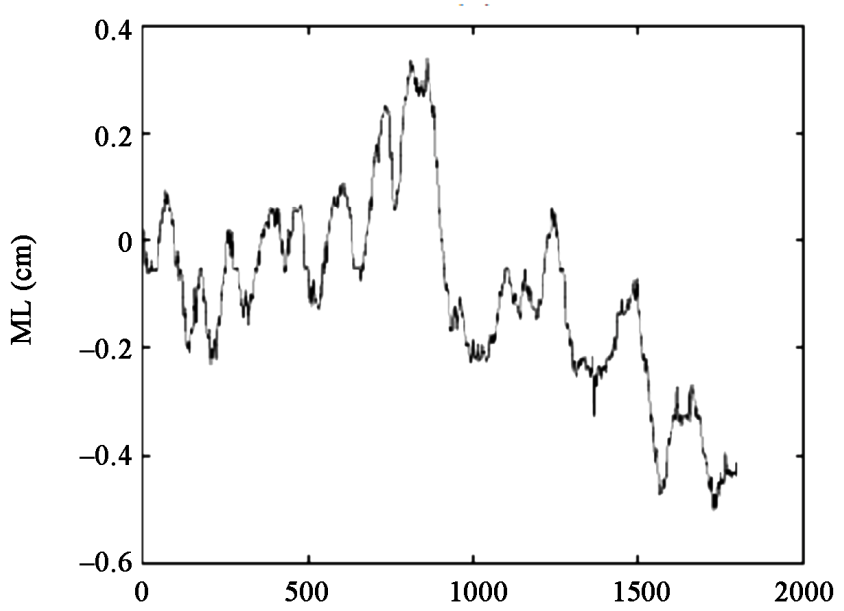

(b)

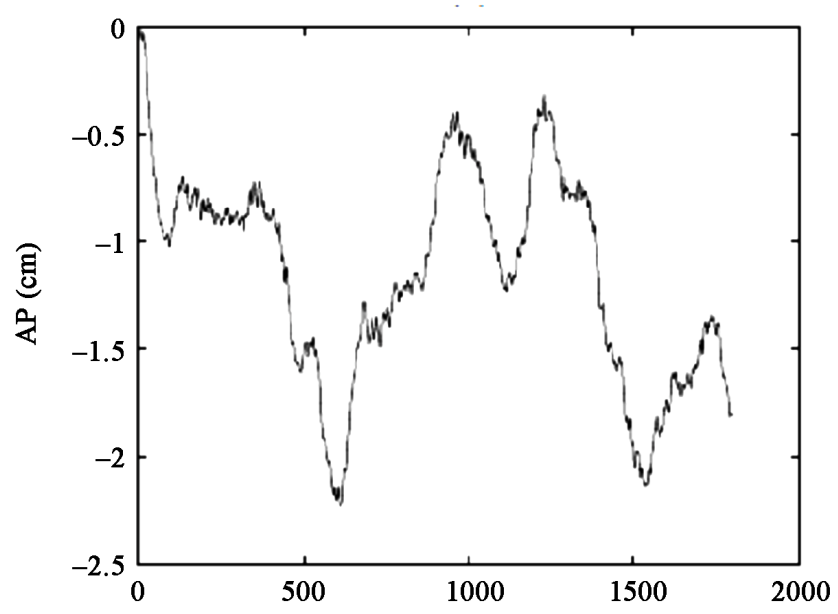

(c)

Figure 1. Displacement of the CG in (a) the horizontal plane; (b) stabilogram in mediolateral (ML) direction; and (c) stabilogram in anteroposterior (AP) displacement. 


\section{METHODS}

\section{1. mPCA Decomposition}

The stabilogram is known to be non-stationary signal, produced by a nonlinear system. It can be considered as a superposition of many signals having different characteristics [8]. These signals can be differentiated by temporal and dimensional characteristics.

The mPCA decomposition requires two steps. In the first step, the signal is decomposed according to a determinist component having a low frequency oscillation (i.e. trend and rambling) and a chaotic signal (i.e. trembling). This is performed thanks to a temporal estimation of the signal, followed by a representation in phase space, then a projection on the first main axes.

In the second step, a polynomial approximation is achieved in order to separate the trend and the rambling. Consequently, the following three components are obtained (Figure 2):

- $\quad$ Trend: the displacement of the principal segment of the considered body;

- Rambling around trend: characterized by a low frequency and a determinist oscillations;

- Trembling around rambling: this signal presents a complex structure having a chaotic nature.

\subsection{Discrete Wavelet Decomposition}

As it is well known, wavelet transform method has the advantage of analyzing signals in a multi-scale manner by varying the scale coefficient (representing frequency) [19].

The wavelet function is defined at scale $a$ and location $b$ as:

$$
\psi_{a, b}(t)=\frac{1}{\sqrt{a}} \psi\left(\frac{t-b}{a}\right)
$$

$\psi_{a, b}(t)$ is also known as "child wavelets" and are derived from a basis function referred to as the "mother wavelet", $\psi(t)[20]$.
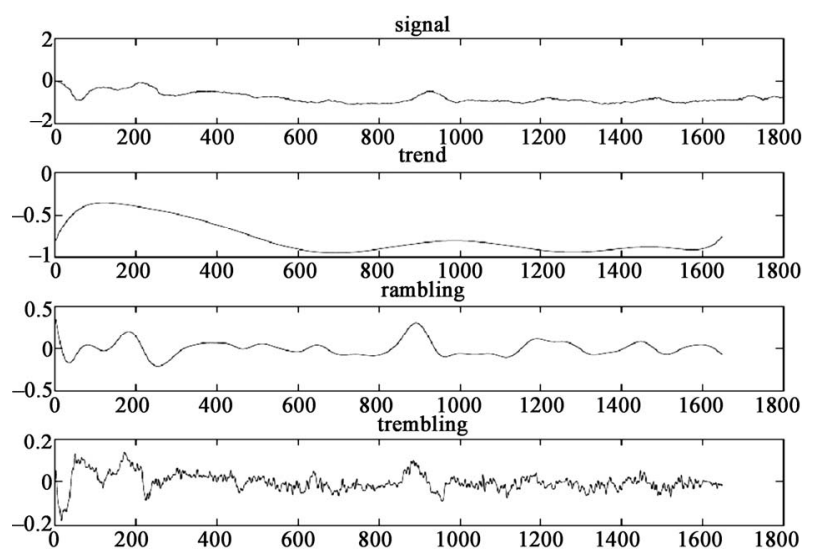

Figure 2. mPCA stabilogram decomposition.
The wavelet transform is given by:

$$
T(a, b)=\int_{-\infty}^{\infty} x(t) \psi_{a, b}^{*}(t) \mathrm{d} t
$$

where $x(t)$ is the time series data and $T(a, b)$ is the "wavelet coefficient" (WC) at timescale $a$ and time instant $b$ [19].

In this study, Daubechies (db2) wavelet function is used to decompose the stabilogram. Three decomposition levels are considered (Figure 3).

\subsection{Parameter Calculation}

The phase is considered as a relevant parameter. However, it cannot be always defined for the complexes signals like stabilogram [6-7]. This is displayed by the visualization of the trajectory in the complex plan of the analytic signal $z(t)$ defined as:

$$
z(t)=s(t)+i \cdot h(t)
$$

where $s(t)$ is the original signal and $h(t)$ is the Hilbert transform of the signal $s(t)$. It is defined as:

$$
h(t)=\frac{1}{\pi} \mathrm{P} \cdot \mathrm{V} \int_{+\infty}^{-\infty} \frac{s(\tau)}{t-\tau} \mathrm{d} \tau
$$

where $\mathrm{P} \cdot \mathrm{V}$ is the Cauchy principle value [6 ].

As shown in Figure 4, one can notice that the trajectory in the complex plan doesn't show a unique rotation center but a multiplicity of centers [6-7]. Consequently, the phase cannot be defined.

Furthermore, the analytic signal can be expressed as:

$$
z(t)=a(t) \cdot \mathrm{e}^{i \cdot(t)}
$$

where $a(t)$ is the amplitude of $z(t)$ and

$$
(t)=\arctan \left(\frac{h(t)}{s(t)}\right) \text { is the instantaneous phase. }
$$

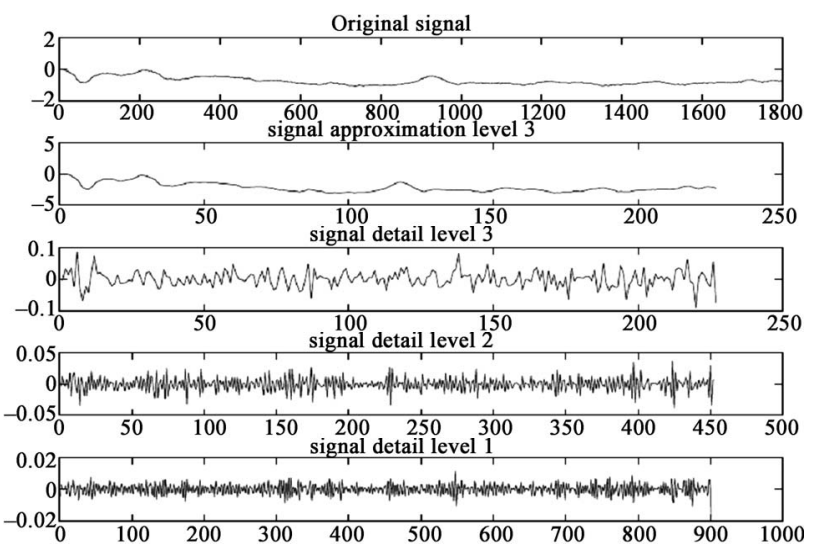

Figure 3. Wavelet stabilogram decomposition. 


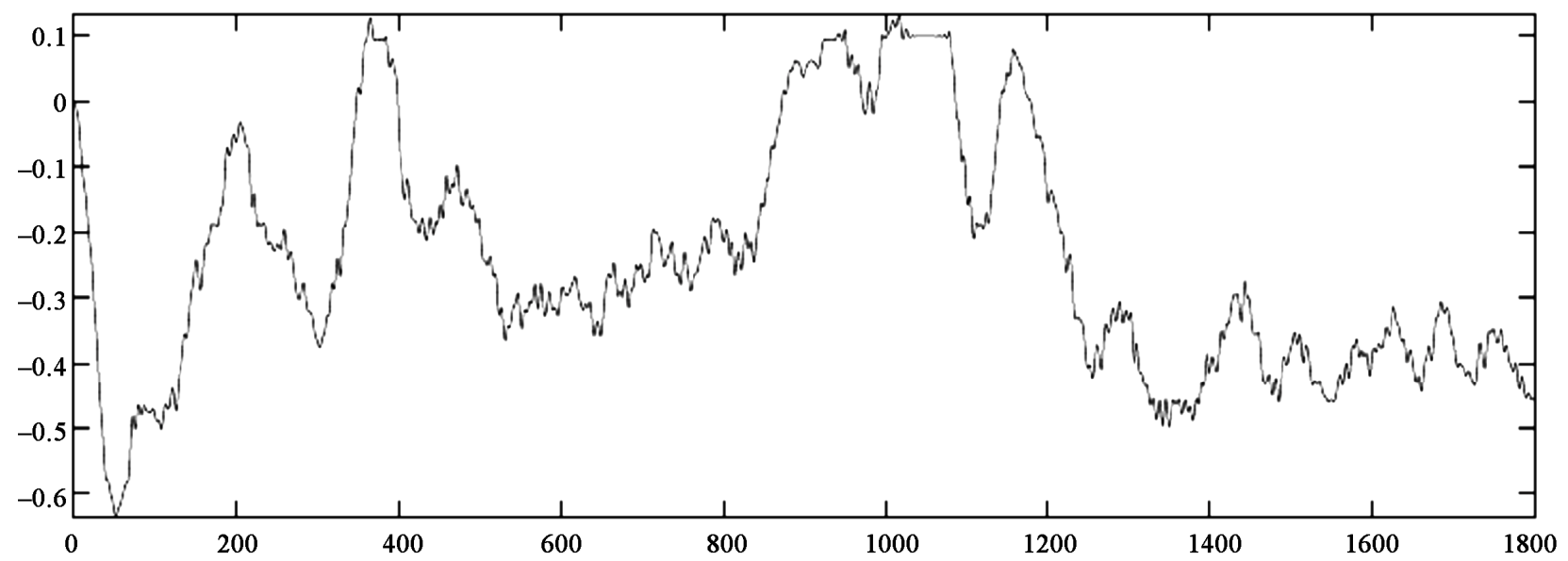

(a)
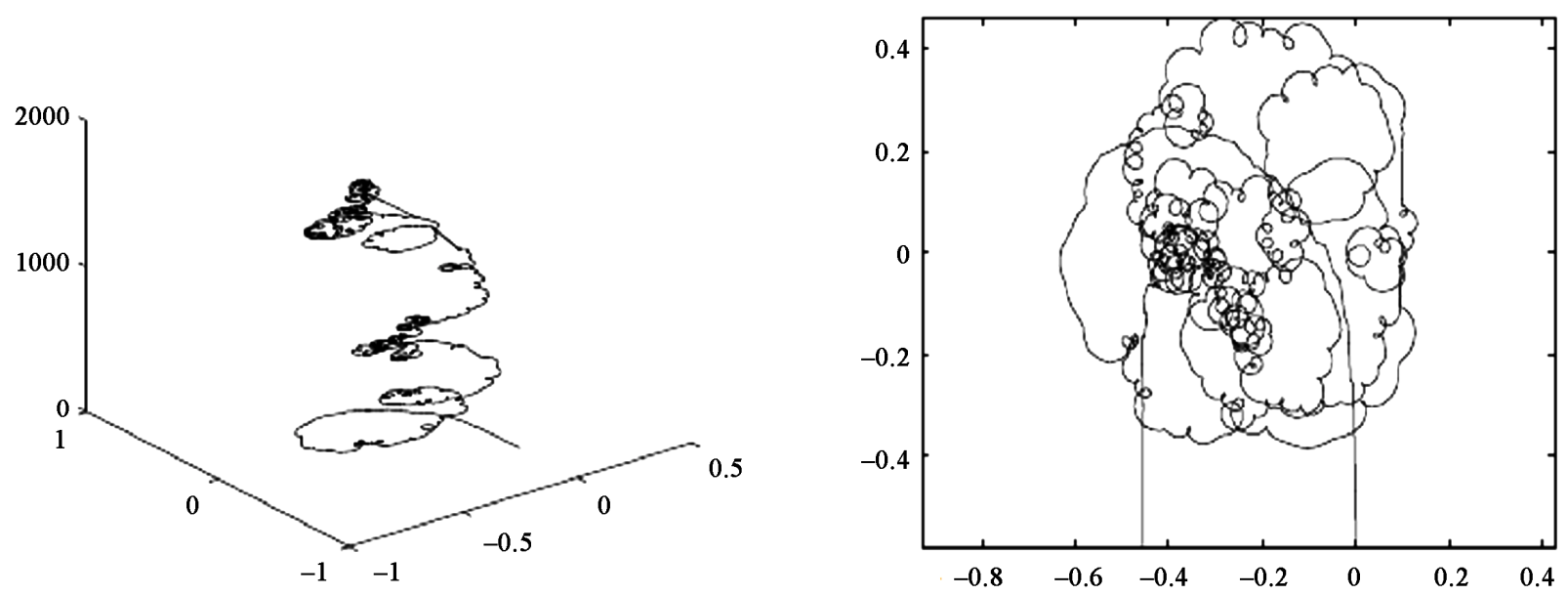

(b)

(c)

Figure 4. (a) Trace of a stabilogram $s(t)$; (b) trajectory in the complex plan $(s, h, t)$; (c) projection in the plan $(s, h)$ highlighting multiple rotation centers.

In order to use the unique definition of instantaneous phase, we have to use a signal having a complex plan trajectory with a unique rotation center.

The visualization of the trajectory in the complex plan of the trembling (either rambling) resulting from mPCA decomposition highlights a unique rotation center (respectively Figures $\mathbf{5}$ and $\mathbf{6}$ ).

This property is similar to that obtained for detail signals resulting from wavelet decomposition (Figures 7, 8 and 9).

Based on the property of having a unique rotation centre from the trembling and rambling trajectory from detail wavelet coefficients trajectory, a specific parameter is defined which consider the area of the circle in which $95 \%$ of the data points are located [10].

This parameter is calculated for AP and ML directions, for the four measures situations (PE YO, PS YO, PS_YF, PE_YF) and for each of these components: trembling and rambling resulting from mCPA decomposition and $\mathrm{cd} 1, \mathrm{~cd} 2, \mathrm{~cd} 3$ resulting from wavelet decom- position. So, for each subject we calculate the mean of all stabilogram area's values. For this purpose, ANOVA was used to compare results between conditions, age categories and gender categories.

\section{RESULTS AND DISCUSSION}

\subsection{Visual Entries Effects}

As shown in previous studies, the lack of visual information causes degradation in the human balance. In fact, when considering closed eyes, the human posture is less stable in comparison to the case of opened eyes (postural Romberg ) $[6,19,20]$.

When using mCPA decomposition, the area's value for rambling was greater for closed eyes (YF) than for opened eyes (YO) and this is the case for both situations PE and PS and both directions ML and AP (Table 1). This increase is indicative of impairment in the stability with (YF) than with (YO). 


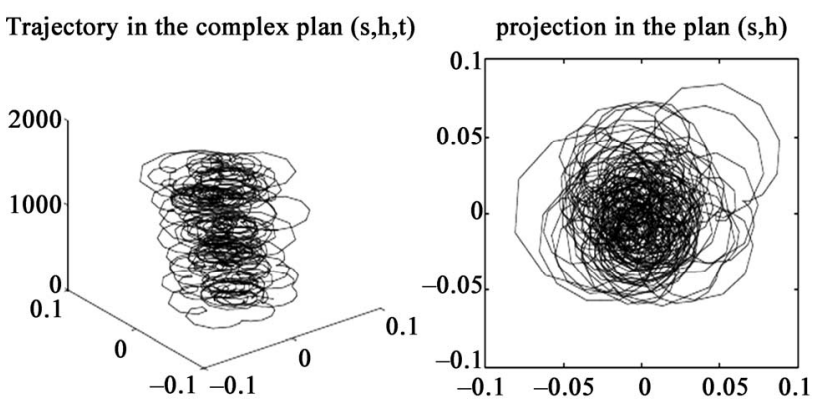

Figure 5. Trajectory in the complex plan $(s, h, t)$ and projection in the plan $(s, h)$ related to trembling, highlighting a unique rotation center.

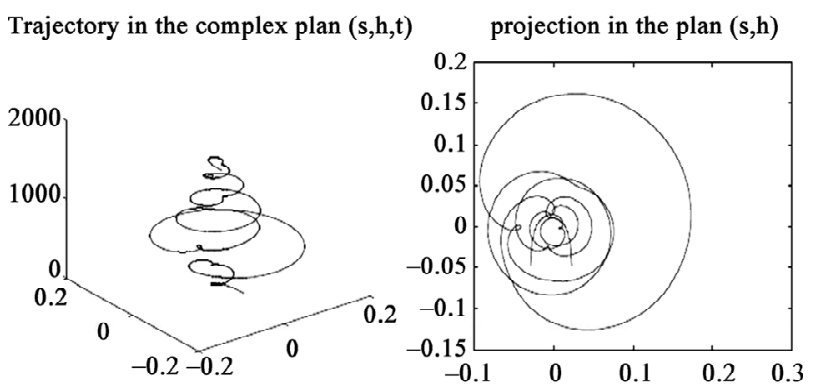

Figure 6. Trajectory in the complex plan $(s, h, t)$ and projection in the plan $(s, h)$ related to rambling, highlighting a unique rotation center.

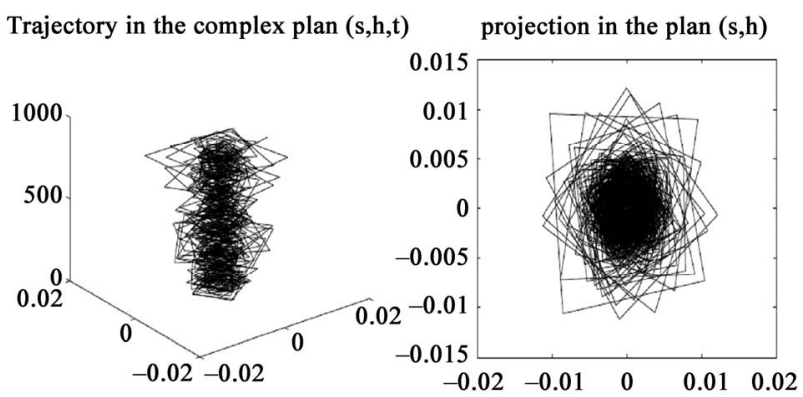

Figure 7. Trajectory in the complex plan $(s, h, t)$ and projection in the plan $(s, h)$ related to $c d 1$, highlighting a unique rotation center.

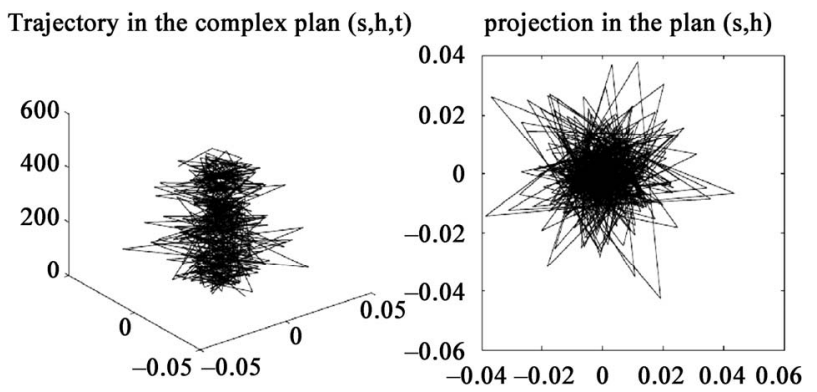

Figure 8. Trajectory in the complex plan $(s, h, t)$ and projection in the plan $(s, h)$ related to $\mathrm{cd} 2$, highlighting a unique rotation center.

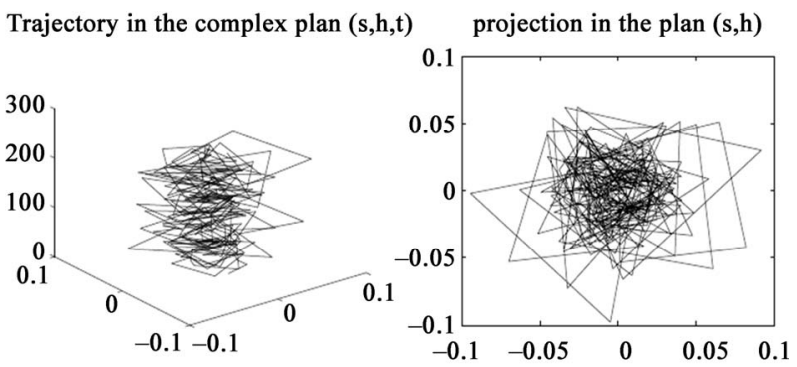

Figure 9. Trajectory in the complex plan $(s, h, t)$ and projection in the plan $(s, h)$ related to $\mathrm{cd} 3$, highlighting a unique rotation center.

However, when using wavelet decomposition, this is not clearly noticeable from $\mathrm{cd} 1, \mathrm{~cd} 2, \mathrm{~cd} 3$ (Table 2).

\subsection{Directional Specificity Effects}

The direction (AP or ML) has an effect on the postural stability based on mCPA decomposition, the areas values in ML direction are greater than in AP direction (especially with rambling) (Table 1). So, as the visual target is in front of the subject standing upright for the measures, the AP is the direction of their eyesight. So, subjects can better control their stability in AP direction. This result is coherent with some previous studies showing that in the direction of head and gazes, subjects can better maintain their stability [21-23].

In fact, if we consider the area values in AP direction, it is clear that with the situations of outspread feet (PE_YO and PE_YF), areas values are very poor (Table 1). This reflects a high ability to maintain equilibrium in AP direction with outspread feet [24]. When considering wavelet decomposition $(\mathrm{cd} 1, \mathrm{~cd} 2, \mathrm{~cd} 3)$ and as seen from the previous results, area values are greater in ML than in AP direction for all situations (Table 2).

Table 1. The average mean values of surface for all subjects for the 4 situations (PE_YF, PE_YO, PS YF and PS YO) for $\mathrm{AP}$ and ML displacement for rambling and for trembling.

\begin{tabular}{ccccc}
\hline & \multicolumn{2}{c}{ rambling } & \multicolumn{2}{c}{ trembling } \\
\cline { 2 - 5 } & ML & AP & ML & AP \\
\hline PE_YF & 1.53408768 & 0.0842523 & 0.64587478 & 0.67442571 \\
PE_YO & 0.94681972 & 0.06734606 & 0.66946591 & 0.67179009 \\
PS_YF & 1.46233659 & 0.81462864 & 0.68186796 & 0.66420952 \\
PS_YO & 0.94070923 & 0.49604842 & 0.6582051 & 0.6592684 \\
P & 0.2034 & $0.0206^{*}$ & $9.0200 \mathrm{e}-005^{* * *}$ & $0^{* * *}$ \\
\hline
\end{tabular}

The asterisk denotes significant differences ${ }^{*} P<0.05 ;{ }^{* *} P<0.01 ;{ }^{* * *} P<$ 0.001 between the 4 situations. 


\subsection{Age Effects}

The healthy subjects are divided into two groups according to their ages: control subject's mean age is $22.5 \pm$ $2.5 \mathrm{y}$ and adult subject's mean age is $34.5 \pm 7.5 \mathrm{y}$.

Based on mPCA decomposition and when we consider the rambling, one can notice that there is a significant difference in area values between groups whatever are the situations, including for AP and ML (Table 3). One can notice too that, the areas are greater for adult subjects than for young subjects.

This increase in the values of area with age can be indicative of degradation in the stability due to age effect.
This result is in agreement with previous studies showing that stability decreases with age: more aged less stable [25-27].

However, this is in contradiction with the result related to trembling showing that area's values for Control are greater than adult (Table 3). This indicates that the control subjects group is less stable than adult group. Consequently, in such a case we conclude that the mPCA is performant, mainly when considering rambling.

When considering wavelet decomposition, results show that for cd1, cd2 and cd3 values (Table 4), there is no significant observation related to the age.

Table 2. The average mean values of surface for all subjects for the 4 situations (PE_YF, PE_YO, PS_YF and PS_YO) for AP and ML displacement; (a) for cd1; (b) for cd2; (c) for cd3.

\begin{tabular}{|c|c|c|c|c|c|c|}
\hline & \multicolumn{2}{|c|}{ cd1 } & \multicolumn{2}{|c|}{ cd2 } & \multicolumn{2}{|c|}{ cd3 } \\
\hline & ML & AP & ML & AP & ML & AP \\
\hline PE_YF & 0.00039101 & 0.00015779 & 0.0060499 & 0.00250787 & 0.02664542 & 0.01034699 \\
\hline PE_YO & 0.00040679 & 0.00015265 & 0.00613421 & 0.00234226 & 0.02874592 & 0.0109055 \\
\hline PS_YF & 0.0004069 & 0.00017734 & 0.00628928 & 0.00269899 & 0.02982301 & 0.01296493 \\
\hline PS_YO & 0.00038226 & 0.00017001 & 0.00598332 & 0.00301738 & 0.02691371 & 0.01166842 \\
\hline$P$ & 0.9230 & $7.9197 \mathrm{e}-004^{* * *}$ & 0.9774 & 0.2402 & 0.8790 & $0.0152^{*}$ \\
\hline
\end{tabular}

The asterisk denotes significant differences ${ }^{*} P<0.05 ;{ }^{* *} P<0.01 ;{ }^{* * *} P<0.001$ between the 4 situations.

Table 3. The average mean values of surface for control and adult subjects for the 4 situations (PE_YF, PE_YO, PS_YF and PS_YO) for rambling in ML displacement; rambling in AP displacement; trembling in ML displacement and trembling in AP displacement.

\begin{tabular}{clcccc}
\hline & & \multicolumn{2}{c}{ rambling } & & trembling \\
\cline { 3 - 6 } Control & & ML & AP & ML & AP \\
\hline & PE_YF & 0.91119186 & 0.05390268 & 0.63572094 & 0.70079239 \\
& PE_YO & 0.46550596 & 0.05128447 & 0.68981477 & 0.68492384 \\
& PS_YF & 1.12265401 & 0.81426074 & 0.68057418 & 0.66607558 \\
adult & PS_YO & 0.57720382 & 0.49154332 & 0.6749946 & 0.66485724 \\
& PE_YF & 2.10906845 & 0.11226733 & 0.50463563 & 0.03801451 \\
& PE_YO & 1.39110934 & 0.08217215 & 0.9293242 & 0.09704093 \\
& PS_YF & 1.77588974 & 0.81496825 & 0.6482783 & 0.20751091 \\
& PS_YO & 1.27625268 & 0.50020697 & 0.51649207 & 0.15789706 \\
& PE_YF & 0.8440 & 0.9066 & 0.1819 & 0.7960 \\
& PE_YO & 0.6251 & 0.9314 & 0.6403 & 0.8034 \\
& PS_YF & 0.8618 & 0.4298 & 0.2564 & 0.4541 \\
\hline
\end{tabular}

The asterisk denotes significant differences ${ }^{*} P<0.05 ;{ }^{* *} P<0.01 ;{ }^{* * *} P<0.001$ between the Control and adult. 
Consequently, in order discriminate the age one should apply the mPCA on rambling which is not the case when dealing with wavelets. Moreover, neither the mPCA nor wavelets can provide significant results when considering the trembling.

From the literature [25-28], one can report that the stability of an individual deceases after 60 years old. When using the mPCA on rambling, two groups can be distinguished which is particularly interesting and promising.

\subsection{Gender Effects}

The healthy subjects are now divided into two groups according to their gender. Female subject's (mean age is $24.5 \pm 5.5 \mathrm{y}$ and male subject's (mean age is $31 \pm 11 \mathrm{y}$ ).

For trembling resulted using mPCA decomposition, results show that there is no significant correlations related to the gender (Table 5). This is in agreement with many studies that failed to find a significant correlation for subjects within in the range $20-49$ y $[19,29]$.

Table 4. The average mean values of surface for control and adult subjects for the 4 situations (PE_YF, PE_YO, PS_YF and PS_YO) for cd1 in ML displacement; cd1 in AP displacement; cd2 in ML displacement; cd2 in AP displacement; cd3 in ML displacement and $\mathrm{cd} 3$ in AP displacement.

\begin{tabular}{|c|c|c|c|c|c|c|c|}
\hline & & \multicolumn{2}{|c|}{ cd1 } & \multicolumn{2}{|c|}{ cd2 } & \multicolumn{2}{|c|}{ cd3 } \\
\hline & & ML & AP & ML & AP & ML & AP \\
\hline \multirow{4}{*}{ control } & PE_YF & 0.00033999 & 0.00015515 & 0.00508957 & 0.0026089 & 0.0209911 & 0.01032489 \\
\hline & PE_YO & 0.00033609 & 0.00014953 & 0.00492099 & 0.00225385 & 0.01974882 & 0.01001624 \\
\hline & PS_YF & 0.00032508 & 0.00018229 & 0.00482645 & 0.00273241 & 0.02046734 & 0.01280845 \\
\hline & PS_YO & 0.0003267 & 0.00017172 & 0.00490177 & 0.00260945 & 0.02027565 & 0.01166333 \\
\hline \multirow{4}{*}{ adult } & PE_YF & 0.0004381 & 0.00016021 & 0.00693636 & 0.00241461 & 0.03186478 & 0.01036739 \\
\hline & PE_YO & 0.00047205 & 0.00015554 & 0.0072541 & 0.00242386 & 0.03705093 & 0.01172635 \\
\hline & PS_YF & 0.00048244 & 0.00017276 & 0.00763958 & 0.00266814 & 0.03845901 & 0.01310937 \\
\hline & PS_YO & 0.00043355 & 0.00016843 & 0.00698169 & 0.00339393 & 0.03304114 & 0.01167312 \\
\hline \multirow{4}{*}{$\boldsymbol{P}$} & PE_YF & 0.0934 & 0.5975 & 0.0728 & 0.5280 & $0.0362^{*}$ & 0.9666 \\
\hline & PE_YO & $0.0454^{*}$ & 0.5223 & $0.0233^{*}$ & 0.3000 & $0.0181^{*}$ & 0.3514 \\
\hline & PS_YF & $0.0102^{*}$ & 0.2797 & $0.0064^{* *}$ & 0.6595 & $0.0067^{* *}$ & 0.7479 \\
\hline & PS_YO & $0.0374^{*}$ & 0.7358 & $0.0194^{*}$ & 0.3945 & $0.0178^{*}$ & 0.9904 \\
\hline
\end{tabular}

The asterisk denotes significant differences ${ }^{*} P<0.05 ;{ }^{* *} P<0.01 ;{ }^{* * *} P<0.001$ between the Control and adult.

Table 5. The average mean values of surface for female and male subjects for the 4 situations (PE_YF, PE_YO, PS_YF and PS_YO) for rambling in ML displacement; rambling in AP displacement; trembling in ML displacement and trembling in AP displacement.

\begin{tabular}{clcccc}
\hline \multirow{2}{*}{ Female } & \multicolumn{2}{c}{ rambling } & \multicolumn{2}{c}{ trembling } \\
\cline { 3 - 5 } & & ML & AP & ML & AP \\
\hline & PE_YF & 0.67627757 & 0.04937066 & 0.65769619 & 0.6774052 \\
& PE_YO & 0.38120252 & 0.06368456 & 0.6749903 & 0.67972198 \\
& PS_YF & 0.90836733 & 0.68712844 & 0.69408988 & 0.66514428 \\
\multirow{3}{*}{ Male } & PS_YO & 0.58459383 & 0.33275789 & 0.63608973 & 0.65382143 \\
& PE_YF & 1.93776303 & 0.10066719 & 0.64031177 & 0.6730236 \\
& PE_YO & 1.21299252 & 0.06906912 & 0.66686619 & 0.66805743 \\
& PS_YF & 1.723028 & 0.87462874 & 0.67611646 & 0.66376963 \\
& PS_YO & 1.10829294 & 0.57289102 & 0.66861233 & 0.66183168 \\
& PE_YF & 0.3518 & 0.3343 & 0.3468 & 0.7665 \\
& PE_YO & 0.8259 & 0.0581 & 0.5018 & 0.4880 \\
& PS_YF & 0.3740 & 0.1567 & 0.5217 & 0.1152 \\
\hline
\end{tabular}

The asterisk denotes significant differences ${ }^{*} P<0.05 ;{ }^{* *} P<0.01 ;{ }^{* * *} P<0.001$ between Female and Male. 
Table 6. The average mean values of surface for female and male subjects for the 4 situations (PE_YF, PE_YO, PS_YF and PS YO) for cd1 in ML displacement; cd1 in AP displacement; cd2 in ML displacement; cd2 in AP displacement; cd3 in ML displacement and cd3 in AP displacement.

\begin{tabular}{clccccccc}
\hline & & \multicolumn{2}{c}{ cd1 } & \multicolumn{2}{c}{ cd2 } & & cd3 \\
\cline { 3 - 7 } Female & & ML & AP & ML & AP & ML & AP \\
\hline & PE_YF & 0.0003563 & 0.00014479 & 0.00555725 & 0.00259958 & 0.02502128 & 0.00930897 \\
& PE_YO & 0.0003478 & 0.00013833 & 0.00527914 & 0.0021248 & 0.02446188 & 0.00944552 \\
& PS_YF & 0.0003879 & 0.00016902 & 0.00597203 & 0.00258519 & 0.02758855 & 0.01271103 \\
& PS_YO & 0.00035635 & 0.00016018 & 0.00570543 & 0.00247141 & 0.02641215 & 0.0108003 \\
& PE_YF & 0.00040734 & 0.0001639 & 0.00628173 & 0.00246471 & 0.02740972 & 0.01083547 \\
& PE_YO & 0.00043455 & 0.00015939 & 0.0065366 & 0.00244459 & 0.03076193 & 0.01159254 \\
& PS_YF & 0.00041585 & 0.00018125 & 0.00643857 & 0.00275254 & 0.03087452 & 0.01308441 \\
& PS_YO & 0.00039446 & 0.00017463 & 0.0061141 & 0.00327431 & 0.02714973 & 0.01207695 \\
& PE_YF & 0.4263 & 0.0531 & 0.5240 & 0.6833 & 0.6816 & 0.1504 \\
& PE_YO & 0.2469 & $0.0282^{*}$ & 0.2740 & 0.0616 & 0.4468 & 0.2729 \\
\hline & PS_YF & 0.6913 & 0.1928 & 0.6963 & 0.2789 & 0.6688 & 0.7095 \\
& PS_YO & 0.5065 & 0.1585 & 0.6858 & 0.4162 & 0.9043 & 0.1353 \\
\hline
\end{tabular}

The asterisk denotes significant differences ${ }^{*} P<0.05 ;{ }^{* *} P<0.01 ;{ }^{* * *} P<0.001$ between Female and Male.

However, if we consider the results related to rambling (Table 5), male's areas are greater than female's.

This means that females are more stable than males in the range varying between $19 \mathrm{y}$ and $42 \mathrm{y}$.

Using wavelet decomposition, the results show that for ML direction and AP direction (except few values), values related to $\mathrm{cd} 1, \mathrm{~cd} 2$ and $\mathrm{cd} 3$ (Table 6) are greater for males than for females. This means that females are more stable than males. However, it is more suitable to use the features extracted from the rambling using the mPCA, rather using wavelets.

Based on mPCA decomposition (especially on rambling) and wavelet decomposition, results are in agreement with previous studies showing that females are more stable than males [1,2,30,31].

\section{CONCLUSIONS}

In this study, mPCA decomposition has been used to decompose the stabilogram into trend rambling and trembling. On the other hand, wavelet decomposition (db3) has been used to decompose the stabilogram into approximation signal and 3 detail signals (cd1, cd2, cd3).

By analyzing the detail signals, namely trembling and rambling signals in the complex plan, it has been clearly noticed that each of these signals present a unique rotation center. By considering the circle where $95 \%$ of the points are located, its area is evaluated to provide a significant parameter.

The analysis of the parameter (circle area) applied on the mPCA rambling signal, projected on the plan $(s, h)$, provided interesting results that allow to distinguish between the visual entries (opened eyes, closed eyes), the directions AP vs ML, the aging and genders. This parameter can be used in some interesting and promising applications such as biometrics and human stability modeling and simulation.

\section{REFERENCES}

[1] Ekhdahl, C., Jarnlo, G. and Andersson, S. (1989) Standing balance in healthy subjects. Scandinavian Journal of Rehabilitation Medicine, 21, 187-195.

[2] Juntunen, J., Ylikoski, J., Ojala, M., Matikainen, E., Ylikoski, M. and Vaheri, E. (1987) Postural body sway and exposure to high-energy impulse noise. The Lancet, 11, 261-264. doi:10.1016/S0140-6736(87)90840-3

[3] Gagey, P., Agey B. and Webber A. (1995) Entrées du système postural fin. Masson, Paris.

[4] Fournier, R., Deléchelle, E. and Lemoine, J. (2001) Décomposition et analyse du signal stabilométrique. $18 e$ Colloque GRETSI'01, Toulouse, 10-13 September 2001, 7.

[5] Fournier, R., Deléchelle, E. and Lemoine, J. (2002) Méthodes de calibrage d'un système électromagnétique pour l'étude et l'évaluation de mesure posturale. Revue Europeenne de Technologie Biomedicale, 23, 303-315. 
[6] Fournier, R. (2002) Analyse stochastique modale du signal stabilométrique. Application à l'étude de l'équilibre chez l'homme. PhD Thesis, Université Paris, Paris, 12.

[7] Fournier, R., Deléchelle, E. and Lemoine, J. (2004) Stabilogram phase estimation. ISIE'2004 IEEE International Symposium on Industrial Electronics, Ajaccio, 4-7 May 2004.

[8] Carroll, J.P. and Freedman, W. (1993) Nonstationary properties of postural sway, Journal of Biomechanics, 26, 409-416. doi:10.1016/0021-9290(93)90004-X

[9] Amoud, H., Snoussi, H., Hewson, D. and Duchêne, J. (2007) Hilbert-Huang transformation: Application to postural stability analysis. 29th Annual International Conference of the IEEE on Engineering in Medicine and Biology Society, Lyon, 22-26 August 2007, 1562-1565.

[10] Amoud, H., Snoussi H., Hewson, D. and Duchêne, J. (2008) Univariate and bivariate empirical mode decomposition for postural stability analysis. EURASIP Journal on Advances in Signal Processing, 2008, 657391.

[11] Huang, N.E., Shen, Z., Long, SR., M.L.C., Shih, H.H., Zheng, Q.N., Yen, N.C., Tung, C. and Liu, H. (1998) The empirical mode decomposition and the Hilbert spectrum for nonlinear and non-stationary time series analysis, Proceedings of the Royal Society A: Mathematical, Physical and Engineering Sciences, 454, 903-995.

[12] Davis, J.R., Campbell, A.D., Adkin, A.L., Carpenter, M.G. (2009) The relationship between fear of falling and human postural control. Gait Posture, 29, 275-279. doi:10.1016/j.gaitpost.2008.09.006

[13] Uetake, T., Tanaka, H., Shindo M. and Okada, M. (2004) Two new methods applicable to center of pressure swing analysis. Anthropological Science, 112, 187-193. doi:10.1537/ase.00075

[14] Martínez-Ramírez, A., Lecumberri, P., Gómez, M. (2010) Wavelet analysis based on time-frequency information discriminate chronic ankle instability. Clinical Biomechanics, 25, 256-264.

[15] Bardet, M. and Bertrand, P. (2007) Definition, properties and wavelet analysis of multiscale fractional Brownian motion. Fractals, 15, 1-15. doi:10.1142/S0218348X07003356

[16] Bertrand, P., Bardet M., Dabonneville, M. and Mouzat, A. (2001) Automatic determination of the different control mechanisms in upright position by a wavelet method. IEEE Engineering in Medicine and Biology Society, Istambul, 25-28, 1163-1166.

[17] Zhang, H.B. (2007) Use of statistical mechanics methods to assess the effects of localized muscle fatigue on stability during upright stance. PhD Thesis, Virginia State University, Petersburg.

[18] Chagdes, J., Rietdyk, S., Haddad M., Zelaznik, N., Raman, A., Rhea, K., Silver, A., (2009) Multiple timescales in postural dynamics associated with vision and a secondary task are revealed by wavelet analysis. Experimental
Brain Research, 197, 297-310.

doi:10.1007/s00221-009-1915-1

[19] Black, F.O., Wall, C., Rockette, H. and Kitch, R. (1982) Normal subject postural sway during the Romberg Test, American Journal of Otolaryngology, 3, 309-318. doi:10.1016/S0196-0709(82)80002-1

[20] Lê. T.-T. and Kapoula, Z. (2008) Role of ocular convergence in the Romberg quotient. Gait \& Posture, 27, 493-500.

[21] Andrea Berencsi, A., Masami Ishihara, B. and Kuniyasu, I. (2008) The functional role of central and peripheral vision in the control of posture. Gait \& Posture, 27, 493-500.

[22] Balasubramaniam, R., Riley, M. and Turvey, M. (2000) Specificity of postural sway to the demands of a precision task. Gait and Posture, 11, 12-24. doi:10.1016/S0966-6362(99)00051-X

[23] Ivanenko, Y., Grasso, R. and F. Lacquaniti (1999) Effect of gaze on postural responses to neck proprioceptive and vestibular stimulation in humans. Journal of Physiology, 519, 301-314. doi:10.1111/j.1469-7793.1999.03010.x

[24] McIlroy, W.E. and Maki, B.E. (1997) Preferred placement of the feet during quiet stance: Development of a standardized foot placement for balance testing. Clinical Biomechanics, 12, 66-70. doi:10.1016/S0268-0033(96)00040-X

[25] Abrahámova, D. and Hlavačka, F. (2008) Age-related changes of human balnce during quiet stance. Physiological Research, 57, 957-964.

[26] Du Pasquier, R.A., Blanc, Y., Sinnreich, M., Landis, T., Burkhard, P. and Vingerhoets, F.J.G. (2003) The effect of aging on postural stability: A cross sectional and longitudinal study. Clinical Neurophysiology, 33, 213-218. doi:10.1016/j.neucli.2003.09.001

[27] Fujita, T., Nakamura, S., Ohue, M., Fujii, Y., Miyauchi, A., Takagi, Y. and Tsugeno, H. (2005) Effect of age on body sway assessed by computerized posturography. Journal of Bone and Mineral Research, 23, 152-156. doi:10.1007/s00774-004-0554-7

[28] Hytönen, M., Pyykkö I., Aaalto, H. and Strack, J. (1993) Postural control and age. Acta Otolaryngology, 113, 119-122.

[29] Hageman, P.A., Leibowitz, J.M. and Blanke, D. (1995) Age and gender effects on postural control measures. Archives of Physical Medicine and Rehabilitation, 76, 961-965. doi:10.1016/S0003-9993(95)80075-1

[30] Kinney LaPier, L., Liddle, S. and Bain C. (1997) A comparison of static and dynamic standing balance in older men versus women. Physiotherapy Canada, 49, 207-213.

[31] Ojala, M., Matikainen, E., and Juntunen, J. (1998) Posturography and the dizzy patient: A neurological study of 133 patients. Acta Neurologica Scandinavica, 80, 118-122. doi:10.1111/j.1600-0404.1989.tb03852.x 